Draft VERSION JANUARY 28, 2019

\title{
IMPLICATIONS ON SPATIAL MODELS OF INTERSTELLAR GAMMA-RAY INVERSE-COMPTON EMISSION FROM SYNCHROTRON EMISSION STUDIES IN RADIO AND MICROWAVES
}

\author{
E. Orlando ${ }^{1}$ \\ ${ }^{1}$ Kavli Institute for Particle Astrophysics and Cosmology, W.W. Hansen Experimental Physics Laboratory, Stanford University \\ CA 9305, Stanford, USA; \\ eorlando@stanford.edu
}

\begin{abstract}
Cosmic rays interacting with gas and photon fields in the Galaxy produce interstellar gamma-ray emission (IGE), which accounts for almost 50\% of the photons detected at gamma-ray energies. Models of this IGE have to be very accurate for interpreting the high-quality observations by present gamma-ray telescopes, such as Fermi Large Area Telescope (LAT). Standard models of IGE, used as reference models for analyses of the Fermi LAT data, show spatial discrepancies with respect to the data, underlining the necessity of more realistic models. The same $\mathrm{CR}$ electrons that produce the inverse-Compton component of the IGE produce also interstellar synchrotron emission observed in radio and microwave. However, present standard models do not take advantage of results coming from studies of this interstellar synchrotron emission.

Accounting for such results, in this work we show how they affect the calculated spatial maps of the large-scale inverse-Compton component of the IGE, which are usually used in studies of Fermi LAT data.

It is found that these results significantly affect these spatial model maps even at a $60 \%$ level. In particular, propagation models based on synchrotron studies produce a more peaked inverseCompton emission in the inner Galaxy region with respect to the standard models used to analyze Fermi LAT data. The conclusion is that radio and microwave observations can be included in a multifrequency self-consistent approach for a more accurate modeling of the IGE finalized to a physical comprehensive interpretation of gamma-ray data and its present unexplained features. Model parameters are provided, which supply a more realistic basis for high-energy gamma-ray studies.
\end{abstract}

Keywords: gamma rays, cosmic rays, interstellar medium

\section{INTRODUCTION}

The interstellar gamma-ray emission (IGE) produced by Galactic Cosmic Rays (CRs) is a dominant component of the gamma-ray sky from a few tens of $\mathrm{MeV}$ to hundreds of $\mathrm{GeV}$ (e.g. Strong et al. 2004; Abdo et al. 2009; Ackermann et al. 2012). This emission is produced by interactions of CRs with photons and gas in the interstellar medium (Strong et al. 2007). It was detected already in the 70's with the Small Astronomy Satellite (SAS) and COS-B that observed the entire sky above 50 $\mathrm{MeV}$. Since the era of the Compton Gamma-Ray Observatory (CGRO) the IGE has been carefully studied and modeled for one of two reasons: 1) IGE as confusing foreground for many analyses; 2) IGE as a useful tool for understanding CRs and the interstellar medium. Almost $50 \%$ of the photons detected by Fermi Large Area Telescope (LAT: Atwood et al. 2009) is due to this IGE (Ackermann et al. 2012). Nowadays, uncertainties in IGE models are limiting our understanding of the gamma-ray sky, especially for dark matter searches (e.g. Linden et al. 2012; Calore et al. 2015; Carlson et al. 2016; Hooper \& Linden 2016; Ackermann et al. 2017, and reference therein) and for source detection (e.g. Grégoire \& Knödlseder 2013; Brandt \& Kocsis 2015; Bartels et al. 2016; Di Mauro et al. 2016). Indeed, disentangling possible dark matter signals, or low-significance sources, from pure IGE is very challenging and needs realistic models. A detailed study of the IGE from the whole Galaxy was performed on a grid of 128 propagation models (Ackermann et al. 2012) using Fermi LAT data. However, this study used standard reacceleration models only, which neglect synchrotron studies (e.g. Strong et al. 2011). These models investigated different 2D CR source distributions, gas parameters, and propagation halo sizes. Even though all models provide a good agreement with data, two issues were identified. First, no best-fit model 
or a set of models could be identified for the whole sky. However, data prefer a large CR propagation halo size, and additional gas or CRs in the outer Galaxy. Second, many large-scale structures, such as the so-called Fermi Bubbles (Su et al. 2010; Ackermann et al. 2014), the inner Galaxy excess, and the outer Galaxy excess (Abdo et al. 2010; Ackermann et al. 2011) emerged in the observations as excesses over the adopted model. Moreover, many less structured excesses and even many dips with respect to present models are also shown all over the sky in the high-quality data by the Fermi LAT. Such excesses and dips are similar in intensity to the $\mathrm{GeV}$ excess seen in the Galactic center (Ackermann et al. 2017). Usual analyses of the Fermi LAT data are based on IGE model maps from propagation models that are then fitted to the data. Forefront recent studies use alternative methods such as hydrodynamical simulations for the gas component (e.g. Macias et al. 2018, based on Pohl et al. (2008)) and SkyFACT (Sky Factorization with Adaptive Constrained Templates) for modeling the gamma-ray emission with adaptive templates and penalized likelihoods (Storm et al. 2017). However they still rely on inverse-Compton maps from propagation models as input.

Hence, beside their quite good agreement, present models based on CR propagation and interaction codes such as GALPROP (Moskalenko et al. 1998; Strong et al. 2007; Vladimirov et al. 2011; Orlando \& Strong 2013; Moskalenko et al. 2015), are not able to describe in the entire sky the more and more precise observations of the diffuse emission, thereby challenging our understanding of the gamma-ray sky and its features. Improvements of these models, which are still the official reference models for studies of diffuse emission, will impact any research field that makes use of the IGE models, such as detection of sources, searches for dark matter, studies on the extragalactic emission, etc. This underlines the necessity of more accurate and realistic IGE models ${ }^{1}$.

In the meantime, important updates on $\mathrm{CR}$ propagation models from studies of the interstellar synchrotron emission in radio and microwaves (Strong et al. 2011; Orlando \& Strong 2013; Planck Collaboration et al. 2016c; Orlando 2018) have been obtained using the GALPROP code. It is important to note that $\mathrm{CR}$ propagation models based on Ackermann et al. (2012), and still used in most gamma-ray studies, do not include these results. The first result from these studies is the derivation of the 3D Galactic magnetic field (B-field) distribution and

\footnotetext{
1 A different approach for disentangling emission components in gamma rays independently from propagation models is followed for example by Selig et al. (2015).
}

intensity from synchrotron observations in radio and microwaves. Updated B-field models include both largescale ordered and random components, with $3 \mathrm{D}$ spiral disc and halo constituents, and updated B-field intensities. The second result is that standard reacceleration models as in Ackermann et al. (2012) are strongly disfavored by radio observations, preferring no, or very low, reacceleration instead. This is caused by the large amount of secondary electrons and positrons produced by reacceleration models at $\sim \mathrm{GeV}$ energies. This overproduces synchrotron emission below a few tens of $\mathrm{MHz}$ that overestimates synchrotron data (models without reacceleration instead fit well synchrotron data). Despite this issue, reacceleration models have been the standard models for gamma-ray analyses so far. Both results from synchrotron studies significantly affect the spatial distribution of primary and secondary electrons and positrons and their energy losses, and consequently also the spatial distribution of the calculated gammaray Inverse-Compton (IC) emission. Our recent work (Orlando 2018, hereafter O2018) investigates the interstellar emission for the first time simultaneously in radio frequencies and in gamma-ray energies consistently, including constraints from latest CR direct measurements. While the effects on the calculated gamma rays regarding the spectrum were addressed in our previously mentioned work, here we report on the effects on the calculated gamma rays regarding the spatial distribution, i.e. on the maps used for gamma-ray analyses. This study adds on recent investigations on effects of 3D models of source distribution, gas and interstellar radiation field, which affect the modeling of the gamma-ray emission at several percent level (Jóhannesson et al. 2018).

Here we show the spatial effects on IGE IC model maps when accounting for model constraints coming from the study of interstellar synchrotron emission observed in radio and microwave. These constraints significantly affect standard CR propagation models of IC emission and can help in providing insights on some physically unexplained features seen at gamma-ray energies.

\section{METHOD}

\subsection{A multifrequency approach}

One of the major IGE components is the IC emission from CRs electrons and positrons on the optical and infrared interstellar radiation field, and on the cosmic microwave background. Direct observations of this emission is limited by contamination from the other emission components (pion decay emission by CR protons and heavier nuclei on the gas, bremsstralhung emission by $\mathrm{CR}$ electrons and positrons on the gas), in addition to other diffuse emission components, such as the 
extragalactic background and the contribution of unresolved sources. Fortunately, the same CR electrons and positrons responsible for the IGE IC are also responsible for the interstellar emission seen at the opposite wavelengths, in radio and microwaves. In fact, this radio and microwave emission is produced by primary and secondary CR electrons and positrons spiraling in the Galactic B-field. Observations of this synchrotron emission encode information on B-fields, on CR electrons and positrons, and, hence, on propagation models. Our approach, pioneered in O2018, is to use studies of synchrotron emission in a consistent way to obtain information on propagation models used for gamma-ray studies.

\subsection{Significant results of recent synchrotron studies}

We summarize here the state of the art on studies of the interstellar synchrotron emission limited to those results that can affect the modeling of the IC component of the IGE.

Interstellar synchrotron emission is the most prominent diffuse component at low frequencies (below few $\mathrm{GHz}$ ) measured by ground based radio instruments, and it is an important component in microwaves. Observations of the interstellar synchrotron emission in the radio band from a few $\mathrm{MHz}$ to tens of $\mathrm{GHz}$ were used to constrain CRs and propagation models by Strong et al. (2011), finding that models with no reacceleration fit best synchrotron spectral data. This approach was followed by other similar works (Jaffe et al. 2011; Di Bernardo et al. 2013). More recently, Orlando \& Strong (2013) investigated the spectral and spatial distribution of the synchrotron emission in temperature and polarization for the first time in the context of CR propagation models. Various CR source distributions, CR propagation halo sizes, propagation models (e.g. pure diffusion and diffusive-reacceleration models), and 3D B-field formulations were studied against synchrotron observations, highlighting degeneracies among the parameters used in the modeling. Additionally, in radio, as in gamma-ray energies, models with flat CR source distribution and large halo size were preferred. The best propagation model was identified and the best 3D Bfield models and their intensities for ordered and random components were obtained. These models have been used for producing the low frequency foreground component maps released by the Planck Collaboration (Planck Collaboration et al. 2016b).

Because the CRs responsible for the radio emission are also responsible for producing the gamma-ray emission, very recently we took advantage of this property with the aim of constraining CRs and propagation models by looking at the interstellar emission in radio and gamma-ray energies simultaneously (O2018). This approach provides a handle on both sides of the electromagnetic spectrum in understanding CRs, thereby leaving less room to uncertainties. In that work we also updated the B-field intensities for ordered and random components based on synchrotron Planck data (Planck Collaboration et al. 2016a,b), recent reprocessed 408 MHz map (Haslam et al. 1981; Remazeilles et al. 2015), and by using the latest CR measurements with AMS02 (Aguilar et al. 2013, 2014). These B-field parameters are also updated with respect to the extensive work in Planck Collaboration et al. (2016c), which, even though based on GALPROP models, instead used an early CR electron spectrum based on less precise Fermi LAT measurements (Ackermann et al. 2010). By studying the local interstellar spectrum we found (O2018) that both gamma-ray data and radio/microwave data prefer the spectrum of pure diffusion models with respect to standard reacelleration models, confirming earlier results by Strong et al. (2011) using radio/microwave data only. This is due to the high positron density $\sim 1 \mathrm{GeV}$ produced in the usual reacelleration models, that is in tension with the synchrotron spectrum at the low frequencies and also with the gamma-ray spectrum below $\sim 1 \mathrm{GeV}$. Hence, both synchrotron (radio and microwave) spectral data and gamma-ray spectral data challenge standard re-acceleration models, in favor of diffusion models without or with small reacceleration. The goal of this study is to present the outcome in the IC spatial model maps of using: 1) updated B-field models that fit synchrotron data; 2) propagation models with no reacceleration as supported by synchrotron and gammaray spectral data.

\section{MODELING}

This section describes how we build the CR propagation models and associated IC gamma-ray models used in this work.

\subsection{The GALPROP code}

$\mathrm{CR}$ propagation models and associated interstellar emission are computed by using the GALPROP code (see references above). GALPROP is a numerical code for modeling the propagation of CRs in the Galaxy and for calculating the associated diffuse emissions. Other CR propagation codes are, for example, DRAGON (Evoli et al. 2017), PICARD (Kissmann et al. 2015), USINE (Maurin 2018). GALPROP solves the transport equation for all required $\mathrm{CR}$ species, for given $\mathrm{CR}$ source distribution, B-field, gas, and interstellar radiation field models. The propagation equation is solved numerically on a user-defined spatial grid in 2D or in 3D, and energy 
grid. It takes into account diffusion, convection, energy losses, ionization, and diffusive reacceleration processes. Secondary CRs produced by collisions in the gas, and decay of radiative isotopes are included. GALPROP is officially used by the Fermi LAT Collaboration (e.g. Abdo et al. 2009; Ackermann et al. 2012; Acero et al. 2016; Ackermann et al. 2017, and reference therein). Recently, extensions of GALPROP for modeling the Galactic radio and microwave emission have been presented (Orlando \& Strong 2013). Starting with Strong et al. (2011) it has been extended to include calculations of radio temperature, polarization, absorption, and free-free emission, to model the interstellar emission in a consistent way from radio to gamma rays.

\subsection{CR propagation models}

Four CR propagation models are used. All models fit latest CR leptonic and hadronic measurements by Voyager I (Cummings et al. 2016) and by AMS02 (Aguilar et al. 2013, 2014).

Table 1. Model parameters

\begin{tabular}{ccc}
\hline \hline $\begin{array}{c}\text { Model } \\
\text { name }\end{array}$ & $\begin{array}{c}\text { B-field } \\
(\text { ord, ran })^{a}\end{array}$ & $\begin{array}{c}\text { Propagation } \\
\text { model }\end{array}$ \\
\hline PDDE $^{b}$ & updated & no reacceleration \\
& $(2.7,4.9)$ & \\
PDDEBold $^{c}$ & old & no reacceleration \\
& $(-, 5)$ & \\
DRE $^{c}$ & updated & with reacceleration \\
& $(2.7,4.9)$ & \\
DRE_comb $^{c}$ & old & with reacceleration \\
& $(-, 5)$ & \\
\hline
\end{tabular}

$a_{\text {Intensity in }} \mu G$ of the ordered and random component respectively (additional details on B-field parameters are in 3.2.1).

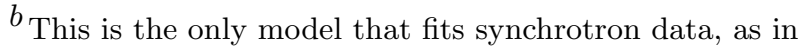
O2018.

$c_{\text {These are similar to the standard models used for }}$ gamma-ray analyses, as in Ackermann et al. (2012). DRE_comb is the most similar to those propagation models.

All the models have the same gas, interstellar radiation field, and CR source distribution. Adopting more complex 3D gas distributions as in Jóhannesson et al. (2018) do not affect our results, because our comparison is made between models with the same gas dis-
Table 2. The table shows the propagation parameters of the models. The description of each parameter can be found in the text.

\begin{tabular}{lcc}
\hline \hline $\begin{array}{l}\text { Propagation } \\
\text { parameters }\end{array}$ & $\begin{array}{c}\text { PDDE \& } \\
\text { PDDEBold }\end{array}$ & $\begin{array}{c}\text { DRE \& } \\
\text { DRE_comb }\end{array}$ \\
\hline & & \\
$\mathrm{D}_{0}{ }^{(a)}\left(\mathrm{cm}^{2} \mathrm{~s}^{-1}\right)$ & 12.3 & 14.6 \\
$\mathrm{D}_{b r}(G V)$ & 4.8 & - \\
$\delta_{1}$ & -0.64 & 0.33 \\
$\delta_{2}$ & 0.58 & - \\
$\mathrm{V}_{\text {Alf }}\left(\mathrm{Km} \mathrm{s}^{-1}\right)$ & - & 42.2 \\
$\mathrm{z}(\mathrm{kpc})$ & 4 & 4 \\
\hline
\end{tabular}

${ }^{a} \mathrm{D}_{x x}=10^{28} \beta D_{0}\left(R / D_{R}\right)^{\delta} \mathrm{cm}^{2} \mathrm{~s}^{-1}$, with $D_{R}=40 \mathrm{GV}$.

tribution. The same consideration applies to different CR source distributions and interstellar radiation field. In our models the typical energy density of the magnetic field is comparable to the total energy density of the photons (starlight, infrared, and CMB). This brings to similar importance of the IC emission and the synchrotron emission in the energy budget of the Milky Way, as found in Strong et al. (2010).

The reference model, PDDE, is the only one fitting synchrotron data. To this model, three models are compared: PDDEBold uses an old B-field that do not fit synchrotron data (spatially and in intensity); DRE does not fit synchrotron spectrum because of the high secondary electron and positron $\mathrm{CR}$ spectrum due to reacceleration processes; DRE_comb combines the old B-field (of PDDEBold), with the high CR electron and positron spectrum (of DRE). DRE_comb has very similar condition of the models used in Ackermann et al. (2012) and still officially and largely adopted for Fermi LAT studies.

Table 1 summarizes the general properties of the models. Additional details on the CR parameters can be found in Table 2 and in O2018, and references therein. Propagation parameters in Table 2 are: $D_{0_{x x}}$, the normalization of the diffusion coefficient at the reference rigidity $D_{R} ; D_{b r}$, the rigidity break where the index of the rigidity can assume different values $\left(\delta_{1}\right.$ and $\left.\delta_{2}\right)$; the Alfven velocity $V_{A l f}$, and the halo size z.

Below we report more details on the four models used.

\subsubsection{PDDE}

This is a pure diffusion model with no reacceleration processes. The propagation parameters are taken as in 
O2018, where the electron spectrum is fitted to AMS02, Voyager I, and synchrotron data. The intensity of the ordered component of the B-field is fitted to the Planck polarization map (Planck Collaboration et al. 2016a,b), while the intensity of the random component is fitted to the $408 \mathrm{MHz}$ map (Haslam et al. 1981; Remazeilles et al. 2015). The B-field formulation is from Sun et al. (2008) and Sun \& Reich (2010) model as used in Orlando \& Strong (2013) and Planck Collaboration et al. (2016c), but with intensities refitted to the synchrotron data, due to the different electron spectrum with respect to the works above. This B-field model contains a disk, a halo, and a toroidal component for the ordered B-field, and it is less complex and sophisticated than Jansson \& Farrar (2012) model. It was also the best model in Orlando \& Strong (2013), used as well in Planck Collaboration et al. (2016c), and it was found Planck Collaboration et al. (2016c) to reasonably reproduce the large-scale Planck synchrotron and dust maps, as well as the Jansson \& Farrar (2012) model.

In more details, the B-field formulation for the random component is a simple exponential law:

$\left.B_{\text {ran }}=B_{0 \text { ran }} \exp \left(-\left(R-R_{\text {sun }}\right) / R_{0 \text { ran }}\right) \exp (-|z|) / z_{0 \text { ran }}\right)$; where $B_{0 \text { ran }}=4.9 \mu G, z_{0 \text { ran }}=4 \mathrm{kpc}$, and $R_{0 \text { ran }}=30 \mathrm{kpc}$. For the ordered disc and halo components we use the B-field formulations as in Sun et al. (2008) and Sun \& Reich (2010). For the disc field we took their ASS model plus reversals in rings (ASS+RING), with $B_{0 \text { disk }}=2.7$ $\mu G$, while for the halo field we took the toroidal component with $B_{0 \text { halo }}=2.7 \mu G$. B-field models are in 3D. Additional details on the PDDE model can be found in O2018.

It is worthy noting that our updated ordered B-field intensity is similar to the intensity of the regular Bfield obtained from rotation measurements (see e.g. Beck 2015, and references therein). This means that the anisotropic, or striated, component of the B-field is negligible or very low with respect to previous works (Jansson \& Farrar 2012; Jaffe et al. 2013; Orlando \& Strong 2013; Ferrière 2013; Planck Collaboration et al. 2016c). This is due to the larger density of electrons measured by AMS02 and Pamela with respect to previous measurements. A dedicated work is in preparation regarding synchrotron studies only, in line with Orlando \& Strong (2013) and Planck Collaboration et al. (2016c).

\subsubsection{PDDEBold}

This is a pure diffusion model with no reacceleration processes. Propagation parameters are similar to O2018 and the PDDE model. The electron spectrum is fitted to AMS02 and Voyager I data. The B-field formulation for the random component follows the same analytic ex- ponential form as in the PDDE, but with different parameter values such as $B_{0 \text { ran }}=5 \mu G, z_{0 \text { ran }}=2 \mathrm{kpc}$, and $R_{0 \mathrm{ran}}=10 \mathrm{kpc}$, as in Ackermann et al. (2012). The intensity of the B-field are not reproducing synchrotron data. The B-field formulation is in $2 \mathrm{D}$, instead of $3 \mathrm{D}$ as in PDDE model, and no ordered component is accounted for, similarly to Ackermann et al. (2012). This model does not fit synchrotron data and it is used in the present work with the PDDE model to present the effect of the B-field only.

\subsection{3. $D R E$}

This is a standard reacceleration model similar to the ones used in Ackermann et al. (2012) for gamma-ray analyses. The propagation parameters are taken as in O2018. The electron spectrum is fitted to AMS02 and Voyager I. The intensity of the ordered component of the B-field is taken as the PDDE model. Propagation parameters are listed in Table 2. This model does not fit synchrotron spectral data and it is used in the present work with the PDDE model to present the effect of reacceleration only.

\subsubsection{DRE_comb}

This is a standard reacceleration model similar to the ones used in Ackermann et al. (2012) for gamma-ray analyses. The electron spectrum is fitted to AMS02 and Voyager I. Differently from PDDE model the B-field intensities were not fitted to synchrotron data. The Bfield formulation is the same as used in Ackermann et al. (2012) and in PDDEBold model. This model does not fit synchrotron spectral data and it is used in the present work with the PDDE model to present the effect of reacceleration and of the B-field combined.

\section{RESULTS ON IC MODEL}

To study the differences regarding the calculated IC emission between a model with no synchrotron constraints (PDDEBold, DRE, and DRE_comb), and a model with synchrotron constraints (PDDE), we report the results of the spatial effects on the calculated IC maps with different: 1) B-field models (Section 4.1); 2) propagation models (with and without reacceleration, Section 4.2); 3) the combination of B-field models and propagation models (Section 4.3).

To verify the effect of including synchrotron constraints we run GALPROP with the propagation models previously described. All-sky model intensity maps of the IC emission are produced in HEALPix format order 7 (Górski et al. 2005) for different energies. For illustration Figure 1 shows the IC emission component for PDDE model for three energies: $30 \mathrm{MeV}, 1 \mathrm{GeV}$, 
and $10 \mathrm{GeV}$. Then, we calculate the difference between the models for a given energy, taking PDDE as reference model. We present the results as all-sky spatial fractional residuals of the calculated IC emission maps between the different models and the reference model. This method of visualization of the differences among models has been extensively applied in the past (e.g. Ackermann et al. 2012). Because here we are interested in the spatial distribution only, the intensity maps of the two different models to be compared are normalized to each other in the entire sky to avoid differences in the normalization of the electron spectra between models. This is important especially for reacceleration models below few $\mathrm{GeV}$, where the density of secondary electron and positrons are large. As illustrative examples, we report results for three given energies $(30 \mathrm{MeV}, 1 \mathrm{GeV}$, and $10 \mathrm{GeV}$ ), which are covered by the Fermi LAT and where the large-scale IC emission is important.

\subsection{Effects of B-field models}

Standard IGE models assume a simple exponential formulation for the B-field, whose intensity and distribution do not fit synchrotron data (spatially or in intensity). PDDEBold model includes a B-field as used in standard models in Ackermann et al. (2012) and following Fermi LAT publications, while PDDE model includes ordered and random 3D B-field components that fit synchrotron observations. Figure 2 shows the total B-field intensity for PDDE (red lines) and PDDEBold (blue lines) models as a function of Galactocentric distance (top) and halo hight (bottom) for a sample of halo hight $(\mathrm{z}=0$ and $\mathrm{z}=2 \mathrm{kpc})$ and Galactocentric radius $(\mathrm{R}=0$ and $\mathrm{R}=8.5 \mathrm{kpc})$. While the B-field intensity for model PDDEBold is larger in the inner Galaxy (in the plane), in general for almost the entire Galaxy PDDE has a much larger and flatter B-field intensity. This is not surprising because this model includes also the ordered component (disk and halo), and because the random component has a larger Galactocentric radius and a larger halo hight. More importantly, PDDE has a much uniform B-field intensity over the sky than PDDEBold, which instead is peaked in the inner Galaxy and drops much faster than the PDDE model in the halo. This different gradient between inner Galaxy and halo reflects on the IC spatial maps.

Figure 3 shows the all-sky spatial fractional residuals of the calculated IC emission between PDDE and PDDEBold model, i.e. (PDDEBold-PDDE)/PDDEBold, for three energies: $30 \mathrm{MeV}, 1 \mathrm{GeV}$, and $10 \mathrm{GeV}$. The only difference between PDDEBold and PDDE is the Bfield. The residuals at all energies exhibit a large-scale trend: the regions outside the inner Galaxy are brighter
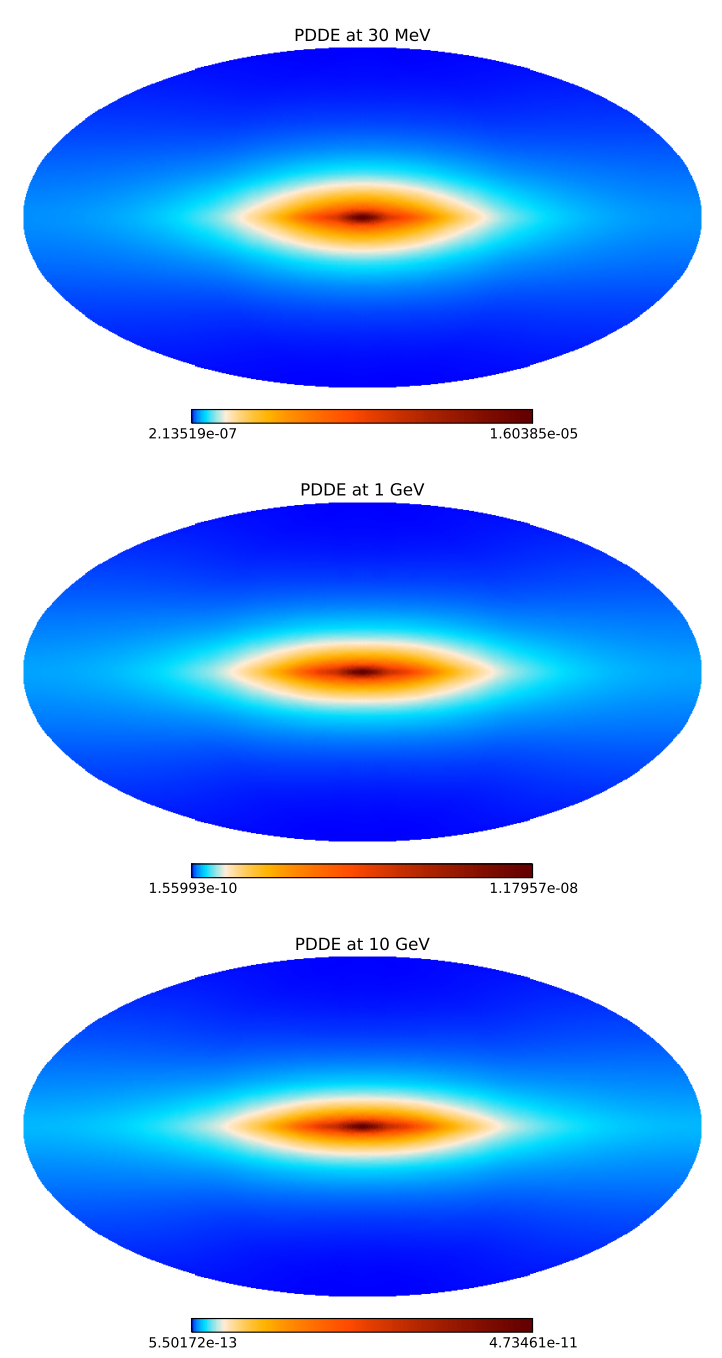

Figure 1. Calculated IC emission for PDDE model at $30 \mathrm{MeV}, 1 \mathrm{GeV}, 10 \mathrm{GeV}$ top to bottom. Maps are in Galactic coordinates with $(1, b)=(0,0)$ at the center of the map. The colorbar indicates the intensity in units of $\mathrm{MeV}^{-1} \mathrm{~cm}^{-2} \mathrm{sr}^{-1} \mathrm{~s}^{-1}$.

for the PDDEBold model than for the PDDE model, with respect to the inner Galaxy region. This is due to the different B-field that produces different energy losses, and hence different distribution of electrons in the sky (close and far to the CR sources). Indeed for almost the entire sky the intensity of the total B-field is larger in the PDDE model than in the PDDEBold model. Electrons loose energies faster with a larger B-field, and produce less IC emission in region outside the inner Galaxy. Since energy losses increase with energy, the differences in the IC emission follow the same trend. In the inner Galaxy the total B-field intensity is reversed, being larger for the PDDEBold than for PDDE. It is interesting to note that the spatial gradient between regions outside the Galactic plane and the 

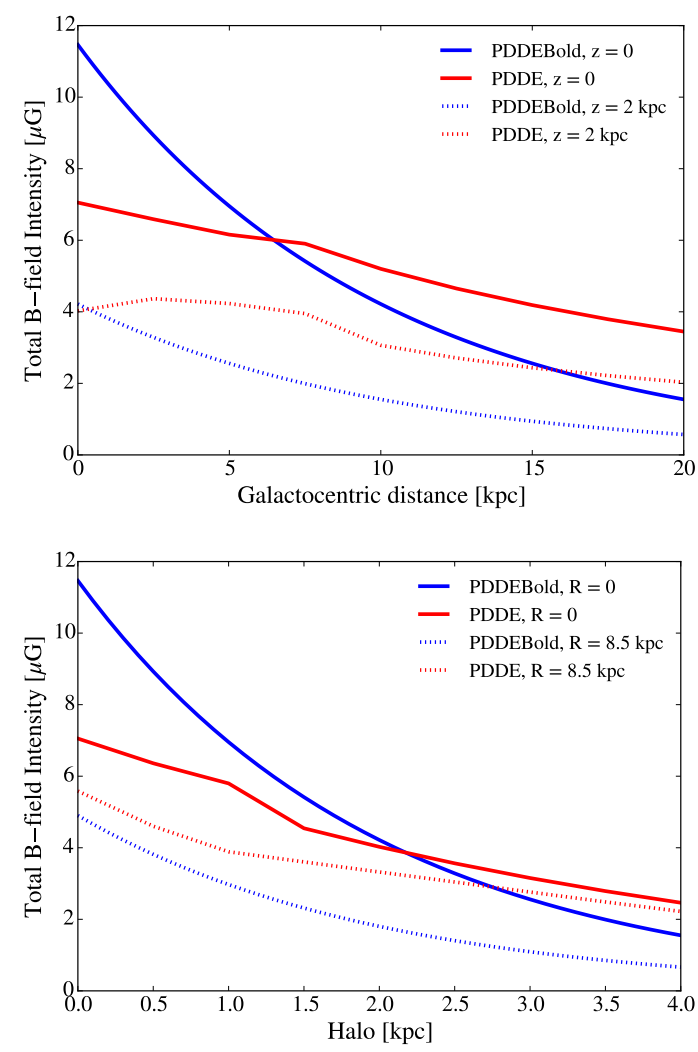

Figure 2. Total B-field intensity for PDDE (red lines) and PDDEBold (blue lines) models. Top: B-field as a function of Galactocentric distance for $\mathrm{z}=0$ (solid line) and $\mathrm{z}=2 \mathrm{kpc}$ (dotted line). Bottom: B-field as a function of halo hight for $\mathrm{R}=0$ (solid line) and $\mathrm{R}$ $=8.5 \mathrm{kpc}$ (dotted line).

inner Galaxy region increases with the energy, being the IC emission for PDDE more peaked in the inner Galaxy region than for PDDEBold. In other words, the IC intensity from the inner Galaxy to the halo decreases faster for PDDE than for PDDEBold, and this is more significant at higher energies. This exactly reflect the trend of the B-field intensity as shown in Figure 2. The extreme intensity values in the calculated IC emission between inner Galaxy and halo reach more than $30 \%$ at $10 \mathrm{GeV}$.

\subsection{Effects of propagation models}

While reaceleration models have been extensively used in gamma-ray and radio data, the all-sky spatial emission of pure diffusion models were used with radio data, and only very recently with gamma-ray data.

Figure 4 shows the all-sky spatial fractional residuals of the calculated IC emission between PDDE and DRE model, i.e. (DRE-PDDE)/DRE, for three energies: 30 $\mathrm{MeV}, 1 \mathrm{GeV}$, and $10 \mathrm{GeV}$. As already noted above, because here we are interested in the spatial distribu-

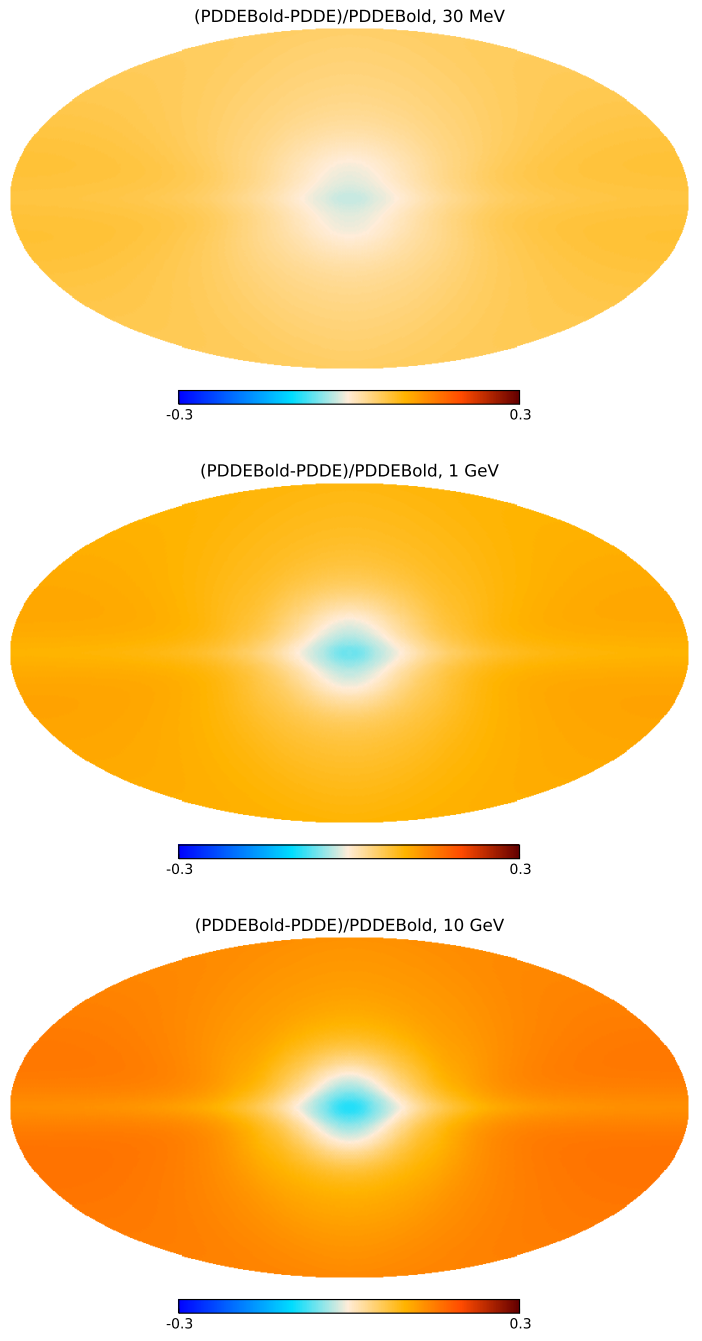

Figure 3. All-sky fractional residuals ((PDDEBoldPDDE)/PDDEBold) show the effects of B-field in the calculation of the IC emission. Residuals are reported at 30 $\mathrm{MeV}, 1 \mathrm{GeV}, 10 \mathrm{GeV}$, top to bottom. Residual maps are in Galactic coordinates with $(1, b)=(0,0)$ at the center of the map. The colorbar ranges from -0.3 to 0.3 .

tion only, the intensity maps of the two different models are normalized to each other in the entire sky to avoid differences in the normalization of the electron spectra between models. This is important especially for reacceleration models below few $\mathrm{GeV}$, where the density of secondary electrons and positrons are large. The spatial difference between PDDE and DRE is given by the different propagation models (reacceleration and pure diffusion models). The B-field is instead the same for the two models. DRE is a standard reacceleration model as used in standard models in Ackermann et al. (2012) and following Fermi LAT publications, while PDDE does not have reacceleration in order to fit synchrotron spectral observations. At the lowest energies (top and middle 

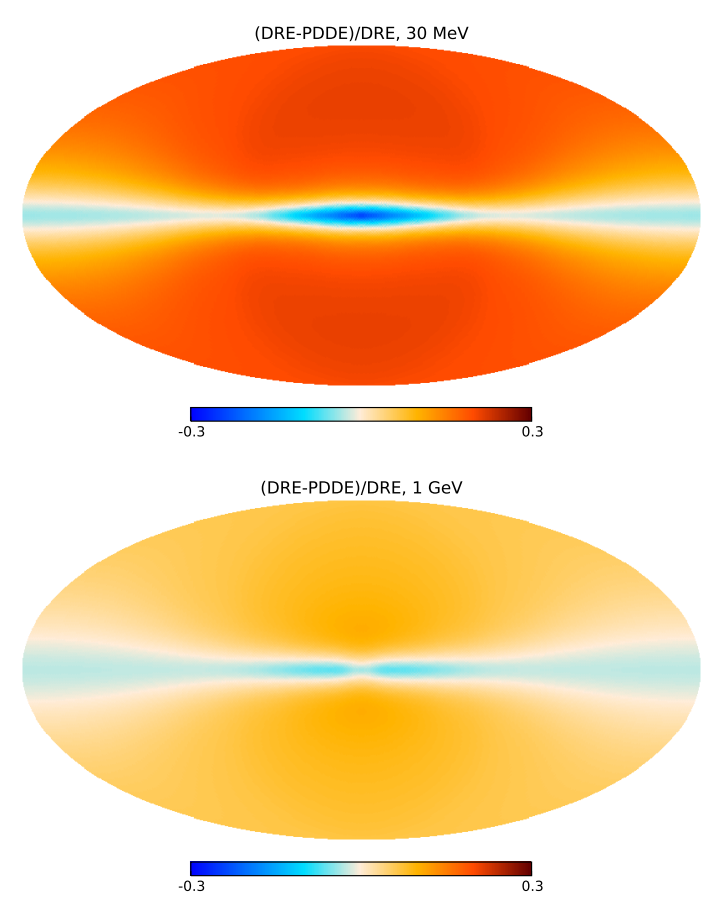

(DRE-PDDE)/DRE, $10 \mathrm{GeV}$

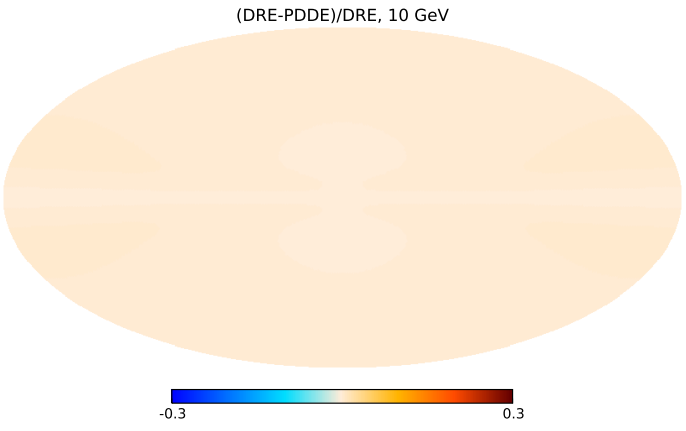

Figure 4. All-sky fractional residuals ((DRE-PDDE)/DRE) show the effects of propagation models in the calculation of the IC emission. Residuals are reported at $30 \mathrm{MeV}, 1$ $\mathrm{GeV}, 10 \mathrm{GeV}$, top to bottom. Residual maps are in Galactic coordinates with $(1, b)=(0,0)$ at the center of the map. The colorbar ranges from -0.3 to 0.3 .

figures) the intensity from the Galactic plane to the halo decreases faster for the PDDE model than for the DRE model. Indeed, the PDDE model has electrons and positrons much closer to the plane, i.e. where CR source are located, than DRE model. The DRE model, instead, has more electrons and positrons in the halo than PDDE model. This is due to reacceleration processes in the DRE model. Indeed, CRs very likely spend a considerable time propagating outside the disc in the extended halo. This produces a similar trend than before: IC emission is much peaked in the inner Galaxy for PDDE than for DRE. It is also interesting to note that the IC emission in the outer Galactocentric radius is significantly brighter for PDDE than for DRE model.
These differences are more notable at the lowest energies (maps at the top), where reacceleration processes have the most effect. Above $10 \mathrm{GeV}$ (map at the bottom) this effect is negligible.

The extreme intensity values in the calculated IC emission between inner Galaxy and halo reach $\sim 60 \%$ at 30 $\mathrm{MeV}$.

\subsection{Effects of B-field and propagation models combined}

Figure 5 shows the all-sky spatial fractional residuals of the calculated IC emission between PDDE and DRE_comb model, i.e. (DRE_comb-PDDE)/DRE_comb, for three energies: $30 \mathrm{MeV}, 1 \mathrm{GeV}$, and $10 \mathrm{GeV}$. The spatial difference between PDDE and DRE_comb is given by the presence and the absence of reacceleration processes and by the different B-field. DRE_comb is a standard reacceleration model as used in standard models in Ackermann et al. (2012) and following Fermi LAT publications, which does not account for synchrotron observations.

In general the combination of the updated B-field and propagation model strengthen the differences in the residuals maps. It is interesting to point out that the PDDE model provides a significantly more peaked IC emission in the inner Galaxy region than DRE_comb model at all energies. In general the extreme intensity values in the calculated IC emission between inner Galaxy and halo for the two models reach the order of $\sim 60 \%$.

\section{DISCUSSIONS AND CONCLUSIONS}

Usual analyses of Fermi LAT data are based on IC model maps from propagation models that are fitted to the data as they are, or rarely they are fitted in Galactocentric rings, as in more recent sophisticated analyses. There is a common consensus about the need of realistic models of the IC emission. Indeed, any advancement in the precision of the IGE models provides a huge impact on our understanding of present gamma-ray observations and can offer insights in describing gammaray features seen with Fermi LAT. Usual propagation model maps adopted in most of the gamma-ray analyses neglect the information coming from interstellar synchrotron radiation in radio and microwaves without taking advantage of them. In particular from synchrotron studies two outcomes are of fundamental importance for models of the IGE IC emission: the knowledge of the B-field (its intensity and spatial distribution), and the information of propagation models. In more detail, the 2D B-field formulation used in standard models for Fermi LAT analyses does not contain any halo and largescale ordered components, which have a 3D formulation. 

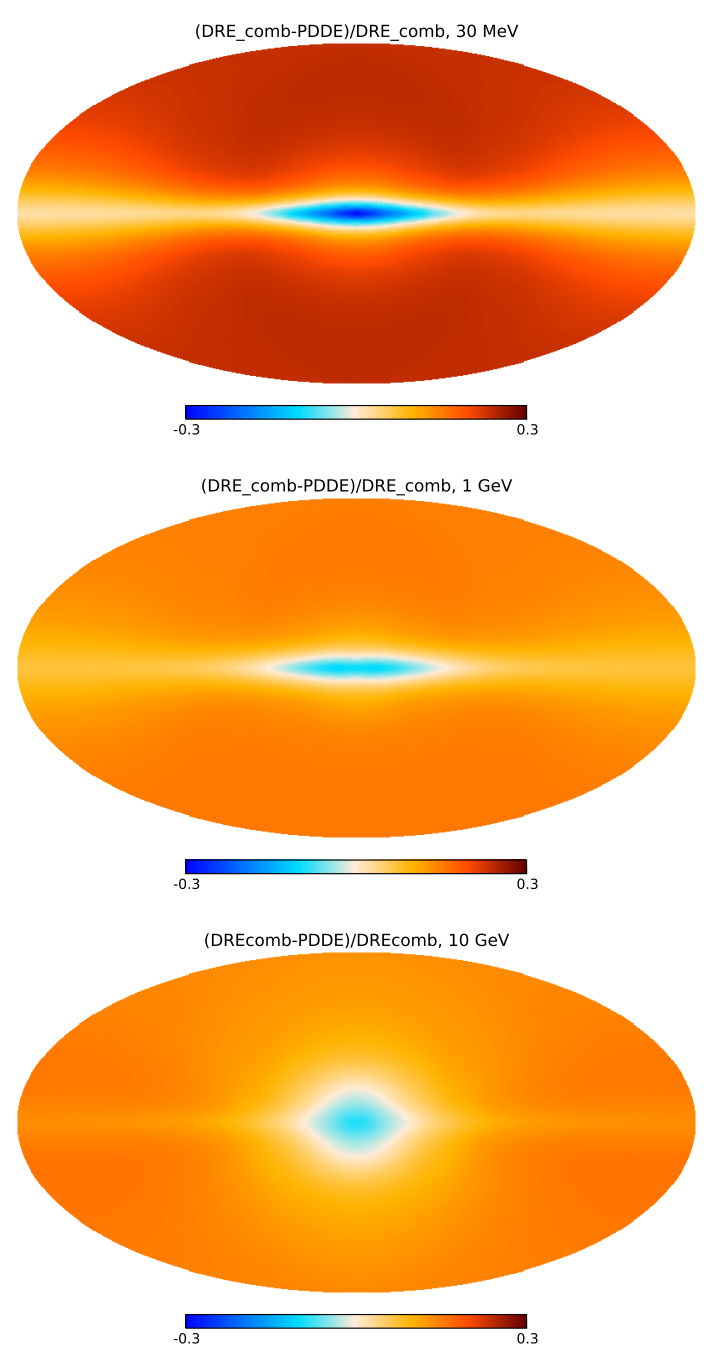

Figure 5. All-sky fractional residuals ((DRE_combPDDE)/DRE_comb) show the effects of propagation models in the calculation of the IC emission. Residuals are reported at $30 \mathrm{MeV}, 1 \mathrm{GeV}, 10 \mathrm{GeV}$, top to bottom. Residual maps are in Galactic coordinates with $(1, b)=(0,0)$ at the center of the map. The colorbar ranges from -0.3 to 0.3

More importantly, the B-field intensity, which is fundamental for calculating propagation and energy losses, is not constrained in those models. In addition, usual IGE models adopted in most of the gamma-ray analyses, are propagation models with reacceleration, which have been found to be strongly disfavored by radio data. This study presents the effects on the IC gamma-ray spatial model maps if results from synchrotron studies are included in the propagation modeling. We investigated the effects on the IC component calculated with the GALPROP propagation code with no reacceleration and with updated 3D B-field models, as supported by synchrotron observations.

By investigating these updated models with models that do not contain such results, we found that the spatial effect in the all-sky IC maps is of the order of $\pm 30 \%$. Such an effect applies to any CR source distribution, gas, and interstellar radiation field model used. Remarkably, the propagation model with a B-field that accounts for synchrotron data produces more peaked IC emission in the inner Galaxy than the standard models used as references for gamma-ray analyses. The difference between the halo and the inner Galaxy reaches even 30\%, increasing with energy. It is interesting to note that a recent analysis of Fermi LAT data based on standard models (Ajello et al. 2016) found an excess of data over models that was best fitted with an enhanced IC component due to enhancements of either the interstellar radiation field or the CR electrons, given their degeneracy. Some other works attribute instead this excess to a population of unresolved sources, to a dark matter component, or to an anisotropic diffusion coefficient. In this work we found that a correct B-field model could accommodate this enhanced IC in the inner Galaxy. In addition, for the same B-field model, propagation models that do not have racceleration produces more IC emission in the plane and especially at large Galactocentric distances than standard models used as reference for gamma-ray analyses. A larger IC emission in the outer plane might be related to the observed excess of data over present IGE models (e.g. Abdo et al. 2010; Ackermann et al. 2011, 2012; Evoli et al. 2012), which would need more gamma rays than predicted at large Galactocentric distances, and that has been attributed to a higher CR density than predicted, additional gas, or to an anisotropic diffusion coefficient.

In conclusion, updated models, as suggested here, provide a more realistic basis for physical interpretation of the gamma-ray data and some features that are not represented by present models, such as the excess in the inner Galaxy and at large Galactocentric radius. We conclude that accurate IGE models can be derived with a multifrequency approach in a self consistent way. Model parameters are provided, which work with synchrotron data and $\mathrm{CR}$ measurements and that can be used for CR propagation models in studies at gamma-ray energies.

This has an important impact for future studies not only with Fermi LAT, but also with possible forthcoming mission at MeV-GeV, such as AMEGO (McEnery 2017), e-ASTROGAM (de Angelis et al. 2018) (and AllSky ASTROGAM), and GAMMA-400 (Ginzburg et al. 2007).

\section{ACKNOWLEDGEMENTS}

E. Orlando acknowledges support from NASA Grants No. NNX16AF27G. 
Useful comments by the anonymous reviewer are acknowledged.
This work makes use of HEALPix ${ }^{2}$ described in Górski et al. (2005).

\section{REFERENCES}

Abdo, A. A., Ackermann, M., Ajello, M., et al. 2009, Physical Review Letters, 103, 251101

Abdo, A. A., Ackermann, M., Ajello, M., et al. 2010, ApJ, 710,133

Acero, F., Ackermann, M., Ajello, M., et al. 2016, ApJS, 223,26

Ackermann, M., Ajello, M., Atwood, W. B., et al. 2010, PhRvD, 82, 092004

Ackermann, M., Ajello, M., Baldini, L., et al. 2011, ApJ, 726,81

Ackermann, M., Ajello, M., Atwood, W. B., et al. 2012, ApJ, 750, 3

Ackermann, M., Albert, A., Atwood, W. B., et al. 2014, ApJ, 793, 64

Ackermann, M., Ajello, M., Albert, A., et al. 2017, ApJ, 840,43

Aguilar, M., Alberti, G., Alpat, B., et al. 2013, Physical Review Letters, 110, 141102

Aguilar, M., Aisa, D., Alvino, A., et al. 2014, Physical Review Letters, 113, 121102

Atwood, W. B., Abdo, A. A., Ackermann, M., et al. 2009, ApJ, 697, 1071

Ajello, M., Albert, A., Atwood, W. B., et al. 2016, ApJ, 819,44

Beck, R. 2015, A\&A Rv, 24, 4

Bartels, R., Krishnamurthy, S., \& Weniger, C. 2016, Physical Review Letters, 116, 051102

Brandt, T. D., \& Kocsis, B. 2015, ApJ, 812, 15

Calore, F., Cholis, I., \& Weniger, C. 2015, JCAP, 3, 038

Carlson, E., Linden, T., \& Profumo, S. 2016, PhRvD, 94, 063504

Cummings, A. C., Stone, E. C., Heikkila, B. C., et al. 2016, ApJ, 831, 18

de Angelis, A., Tatischeff, V., Grenier, I. A., et al. 2018, Journal of High Energy Astrophysics, 19, 1

Di Bernardo, G., Evoli, C., Gaggero, D., Grasso, D., \& Maccione, L. 2013, JCAP, 3, 36

Di Mauro, M., Donato, F., Fornengo, N., \& Vittino, A. 2016, JCAP, 5, 031

Evoli, C., Gaggero, D., Grasso, D., \& Maccione, L. 2012, Physical Review Letters, 108, 211102

Evoli, C., Gaggero, D., Vittino, A., et al. 2017, JCAP, 2, 015

${ }^{2}$ http://healpix.jpl.nasa.gov/
Ferrière, K. 2013, SF2A-2013: Proceedings of the Annual meeting of the French Society of Astronomy and Astrophysics, 13

Ginzburg, V. L., Kaplin, V. A., Karakash, A. I., et al. 2007, Cosmic Research, 45, 449

Górski, K. M., Hivon, E., Banday, A. J., et al. 2005, ApJ, 622,759

Grégoire, T., \& Knödlseder, J. 2013, A\&A, 554, A62

Kissmann, R., Werner, M., Reimer, O., \& Strong, A. W. 2015, Astroparticle Physics, 70, 39

Haslam, C. G. T., Klein, U., Salter, C. J., et al. 1981, A\&A, 100,209

Hooper, D., \& Linden, T. 2016, JCAP, 8, 018

Jaffe, T. R., Banday, A. J., Leahy, J. P., et al. 2011, MNRAS, 416, 1152

Jaffe, T. R., Ferrière, K. M., Banday, A. J., et al. 2013, MNRAS, 431, 683

Jansson, R., \& Farrar, G. R. 2012, ApJL, 761, L11

Jóhannesson, G., Porter, T. A., \& Moskalenko, I. V. 2018, ApJ, 856, 45

Linden, T., Lovegrove, E., \& Profumo, S. 2012, ApJ, 753, 41

Macias, O., Gordon, C., Crocker, R. M., et al. 2018, Nature Astronomy, 2, 387

Maurin, D. 2018, arXiv:1807.02968

McEnery, J. E. 2017, AAS/High Energy Astrophysics Division \#16, 16, 103.13

Moskalenko, I. V., Strong, A. W., \& Reimer, O. 1998, A\&A, 338, L75

Moskalenko, I. V., Jóhannesson, G., Orlando, E., et al. 2015, 34th International Cosmic Ray Conference (ICRC2015), 34, 492

Orlando, E., \& Strong, A. 2013, MNRAS, 436, 2127

Orlando, E. 2018, MNRAS, 475, 2724

Planck Collaboration, Ade, P. A. R., Aghanim, N., et al. 2016, A\&A, 594, A25

Planck Collaboration, Adam, R., Ade, P. A. R., et al. 2016, A\&A, 594, A10

Planck Collaboration, Adam, R., Ade, P. A. R., et al. 2016, A\&A, 596, A103

Pohl, M., Englmaier, P., \& Bissantz, N. 2008, ApJ, 677, 283

Porter, T. A., Jóhannesson, G., \& Moskalenko, I. V. 2017, ApJ, 846, 67

Remazeilles, M., Dickinson, C., Banday, A. J., Bigot-Sazy, M.-A., \& Ghosh, T. 2015, MNRAS, 451, 4311 
Selig, M., Vacca, V., Oppermann, N., \& Enßlin, T. A. 2015, A\&A, 581, A126

Storm, E., Weniger, C., \& Calore, F. 2017, JCAP, 8, 022

Strong, A. W., Moskalenko, I. V., Reimer, O., Digel, S., \& Diehl, R. 2004, A\&A, 422, L47

Strong, A. W., Moskalenko, I. V., \& Ptuskin, V. S. 2007, Annual Review of Nuclear and Particle Science, 57, 285

Strong, A. W., Porter, T. A., Digel, S. W., et al. 2010, ApJL, 722, L58
Strong, A. W., Orlando, E., \& Jaffe, T. R. 2011, A\&A, 534, A54

Su, M., Slatyer, T. R., \& Finkbeiner, D. P. 2010, ApJ, 724, 1044

Sun, X. H., Reich, W., Waelkens, A., \& Enßlin, T. A. 2008, A\&A, 477, 573

Sun, X.-H., \& Reich, W. 2010, Research in Astronomy and Astrophysics, 10, 1287

Vladimirov, A. E., Digel, S. W., Jóhannesson, G., et al. 2011, Computer Physics Communications, 182, 1156 